\title{
A Deep Analysis of Google Net and AlexNet for Lung Cancer Detection
}

\author{
B.Almas , K.Sathesh , S.Rajasekaran
}

\begin{abstract}
Lung cancer is the major cancer that cannot be disregarded intentionally and causes deceased with late healthcare. Now, Computed Tomography(CT) scan allows the doctors to recognize the lung cancer in the beginning of the stage. Majority of cases are tends to be failed in diagnosis of determining the lung cancer eventhough the doctors are experienced, they failed to detect the cancer. Deep learning is the important technique that can be applicable in medical imaging diagnosis. In this paper, the implementation of Convolutional Neural Networks such as GoogleNet (Inception) and AlexNet are analyzed for the lung cancer detection. The cancer images from LIDC-IDRI dataset is used for this research work. The Preprocessed cancer images are trained using GoogleNet and AlexNet to determine the cancer affected part of the lungs. The identification of lung cancer by using GoogLeNet and AlexNet are used for training the network, and image classification. These networks are provided with layered architecture for classification. We have found that AlexNet and GoogLeNet provides the comparable results by including parameters like time, initial learning rate and accuracy.
\end{abstract}

Keywords : AlexNet, Accuracy, Convolutional NeuralNetwork, Diagnosis, GoogleNet, Learning rate

\section{INTRODUCTION}

Lung cancer is the major disease that leads to death in both men and women[1]. Some reports estimated that 9.6 million deaths happened in 2018 due to cancer. Among this, 1.76 million deaths are due to lung cancer[2]. The trace of lung cancer describes the total of $27 \%$ cancer deaths in 2015[3]. In order to overcome the mortality rate, lung nodules need to be observed closely at an early stage. By early detection, the survival rate of the patients suffering from lung cancer can be improved by $50 \%$. Computed Tomography(CT) is the efficient method to diagnosis the diseased part of lungs. It is capable of analyzing three dimensional images on the chest that can be widely used in clinic[4]. Deep learning has a tremendous impact on medical imaging diagnosis for recognizing, characterizing the images. Deep learning not only performs the critical tasks but also improves the

Revised Manuscript Received on December 30, 2019.

* Correspondence Author

B.Almas, Electronics \& Communication Engineering, Madanapalle Institute of Technology and Science, Madanapalle ,Andra Pradesh, India.

Dr.K.Sathesh, Associate Professor, Department of Electronics \&Communication Engineering, Madanapalle Institute of Technology and Science, Madanapalle ,Andra Pradesh, India.

Dr.S.Rajasekaran, Associate Professor, Department of Electronics \&Communication Engineering, Madanapalle Institute of Technology and Science, Madanapalle ,Andra Pradesh, India.

(c) The Authors. Published by Blue Eyes Intelligence Engineering and Sciences Publication (BEIESP). This is an open access article under the CC BY-NC-ND license (http://creativecommons.org/licenses/by-nc-nd/4.0/) performances of CT Scan image detection.Convolutional neural network $(\mathrm{CNN})$ is the important architecture in deep learning. CNN will process and classify the input images to the different categorizes(for example lion, cat, dog). Computers display an input image by an array of pixels. It considers hxwxd based on the persistence of the image where h, w, and d specifies the height, width and dimension.Deep learning $\mathrm{CNN}$ will train the set of input images and performs the testing, by validating every input image by undergoing through several convolutional layers with kernels, maximum pooling, and other layers that are fully connected followed by softmax for the classification of an object.The main layer that concentrates the characteristics from a picture is the convolution layer[5].It safeguards the relationship existing between pixels through learning pictures utilizing little information squares. It involves a numerical activity which takes two information sources, a kernel or channel, and picture matrix. Some of the CNN architectures includes GoogLeNet, AlexNet, VGGNet, and ResNet. This paper describes the comparison between AlexNet and GoogLeNet to determine the accuracy rate applicable in the detection of lung cancer.

\section{PROPOSED ALGORITHMS}

The GoogleNet and AlexNet networks are used for recognizing the lung cancer. The current work is categorized into four steps. Step one - The lung cancer images are obtained from LIDC-IDRI image dataset. Step two- The Preprocessed images are trained in this research work. Step three- The irregular format images are converted into the BMP image format. Step four- The cancer images are trained using GoogleNet and Alexnet network. These networks will train the set of images and provides the validated results with desired accuracy rate.The image dataset is divided into $70 \%$ for Training and $30 \%$ for Validation.

\section{A. GoogLeNet (Inception):}

The GoogleNet uses 3 different dimensional filters( i.e., $1 \mathrm{X} 1,3 \mathrm{X} 3,5 \mathrm{X} 5)$ for the input image and combines the characteristics to get the resultant output. This network is 22 layered and diminish the variables from 60 Million (AlexNet) to 4 million[6]. The $1 \times 1$ convolution with 128 filters is applicable for dimension reduction[7].This network will provides the weight during training and determines required features. 


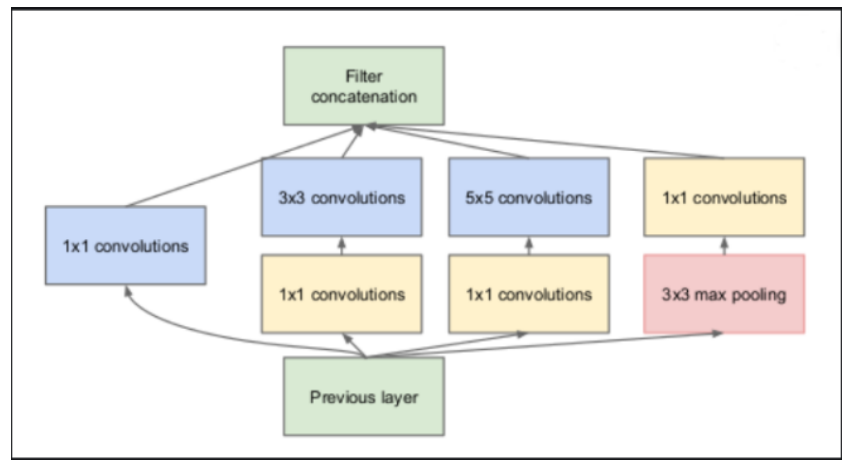

Figure1:Inception with Dimensionality Reduction

The Fig.1 describes the filters( i.e., 1X1, 3X3, 5X5) and pooling layer.

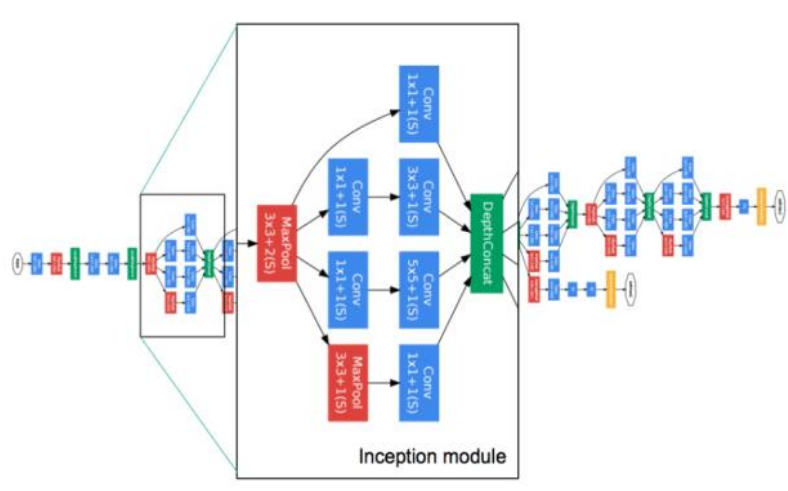

Figure 2: Inception Architecture

Fig. 2 shows the basic block in the network architecture. There are numerous network blocks combined together extensively to obtain the higher accuracy. Transfer Learning depicts the training of images using GoogleNet and AlexNet to identify cancer effected portion of the lungs. The image analysis pretrained network was examined by considering multiple images to classify different problems like benign, malignant tumors[9]. Input images are provided to the network and there is caption of the objects in the images providing the output based on the occurrence of each object path.

Steps in categorizing cancer effected part of lung using GoogleNet :

i) Loading Images: This is the first step in separation of lung cancer image by using GoogleNet. It involves loading the cancer image dataset of morethan 200 images. After loading the images, the process is categorized into two steps. The first is training the $70 \%$ of the images and the second is validation set, validates the remaining $30 \%$ of the images.

ii) Load GoogleNet Network: In this step, the term "analyze-Network" helps in exhibiting the architecture and layer information of the network. The Fig.3 displays the different layers and provides the information on weights, bias in the GoogleNet architecture.

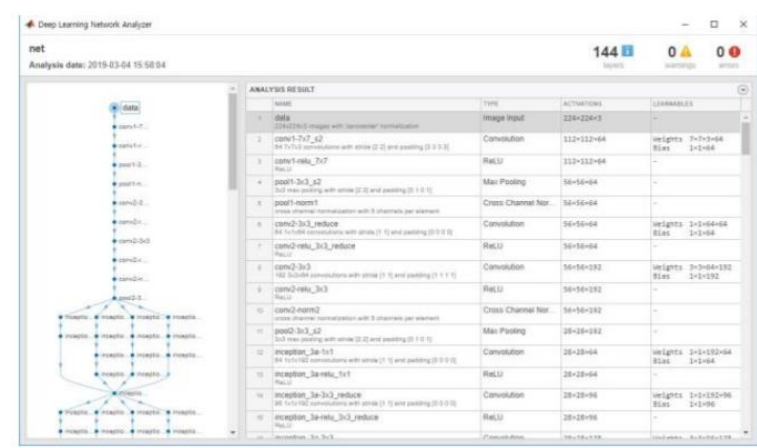

Figure3: GoogLeNet Architecture and Layer information iii) Load Pretrain Network: In this step, different features can be extracted from the input images via through convolution layer which are then examined by classification layer. The features are then integrated by "loss3-classifier" and "output" layers are then collaborated with different probabilistic values. Fig:4 displays the new layers substituting the two layers for retaining GoogleNet.

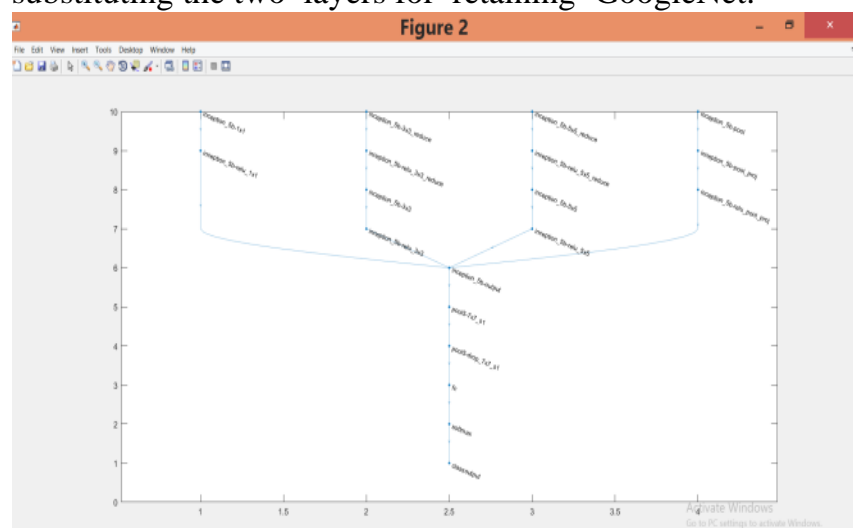

Figure 4: New Layer Graph

iv)Freezing the Basic Layers: In this step, GoogleNet manages the training of new data set by setting initial layers to zero. By freezing the layers, it will enhances the speed of network.

v) Network Training: For training the network, the input images have to be $223 \times 223 \times 3$ in size. During the training, the size of images may change. It accelerates the data by avoiding over-fitting by balancing the memory characteristics of training images. In Fig: 5 the graph displays the accuracy $-99.05 \%$, elapsed time $-46 \mathrm{hrs}$, maximum iterations -1620 , validation frequency -3 iterations, learning rate -0.0001

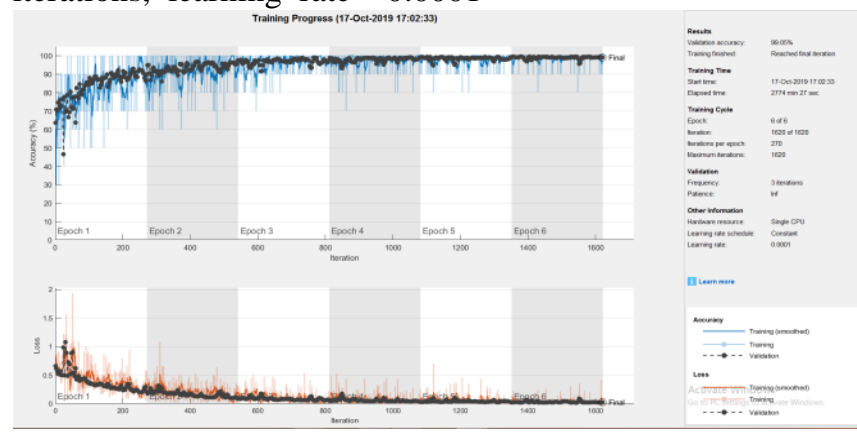

Figure 5: Training Progress 
vi) Image Validation: In the final step validation of images can be specified with different labels that holds various probabilistic values. Fig:6 shows the validated images affected with cancer providing the resultant accuracy.

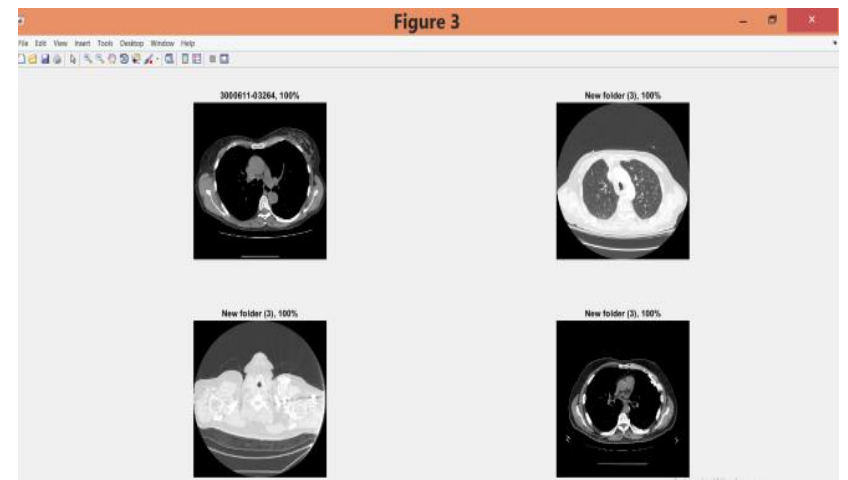

Figure 6: Lung Cancer Detection with Predicted label and Percentage

\section{B.AlexNet:}

AlexNet was proposed by three different authors in 2012 and one among them is Alex Krizhevsky. AlexNet comprises of eight layers:Five convolutional layers and three fully connected(FC) layers with new concepts like Pooling and ReLU activation. The input image size for AlexNet is $227 \mathrm{X} 227 \mathrm{X}$ 3.The convolutional layers use $11 \mathrm{X}$ 11 filters and the pooling uses $3 \times 3$ filters with different strides.

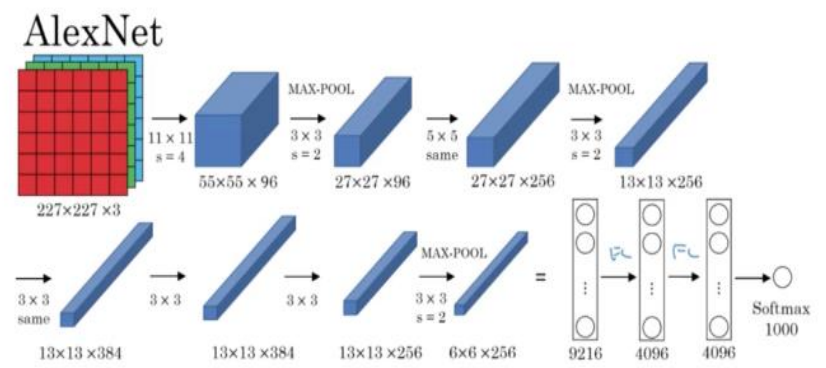

Figure 7: AlexNet Architecture

Steps in categorizing cancer effected part of lung using AlexNet

i) Image Loading: Load LIDC IDRI database which contains 244,527 images of the 1010 cases. It loads 2705 images for training the AlexNet. The function "split Each Label" categorizes $70 \%$ of the images for training and $30 \%$ for validating [10].

ii)Loading AlexNet: Load the AlexNet network which will provide the basic information of the network. Fig.8 shows the flow of AlexNet provided with different weights, bias, padding applicable in convolutional, ReLU and pooling layers.

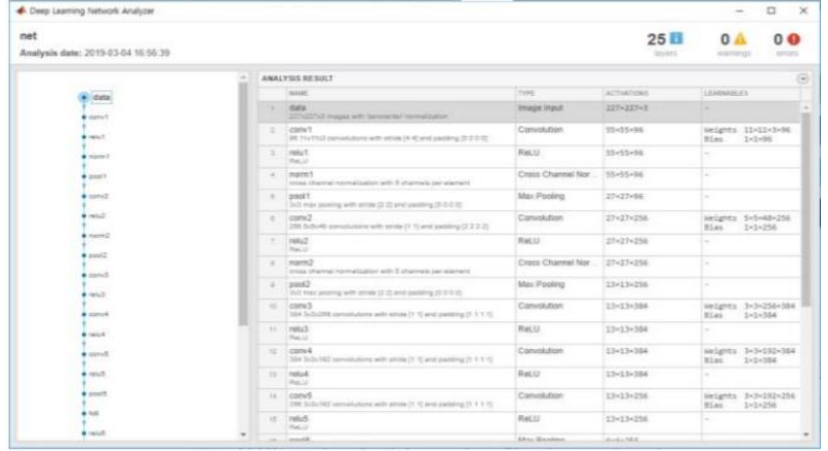

Figure 8: AlexNet Network Architecture and Layer Information

iii)Substitution of the Final Layers: In this step, replacement of convolutional layers with FC layers provides the classification output and softmax layer.

iv)Training Network: During the training, the function 'train-Network (imds, layers, options)' used for classifying the images.The term imds - assembles the input images,layers - clarifies the network configuration, options - like learning rate-0.0001, accuracy-99.91\%,Maximum epochs-6,Validation frequency-3 iterations, Plots, Training progress specified to detect the lung cancer. Fig.9 shows the accuracy plot for AlexNet with options value.

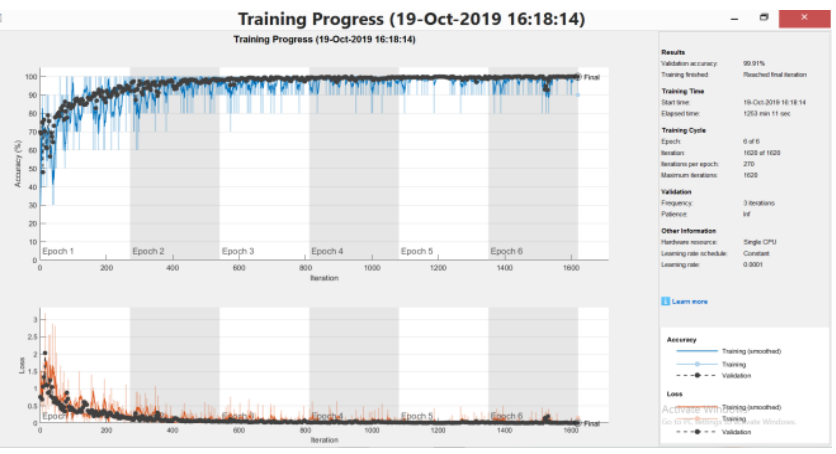

Figure 9: AlexNet Training Progress

v) Image Classification: Final step is to categorize output data by considering validation images and hence accuracy is calculated. Fig.10 displays the validated images with obtained probabilistic values.

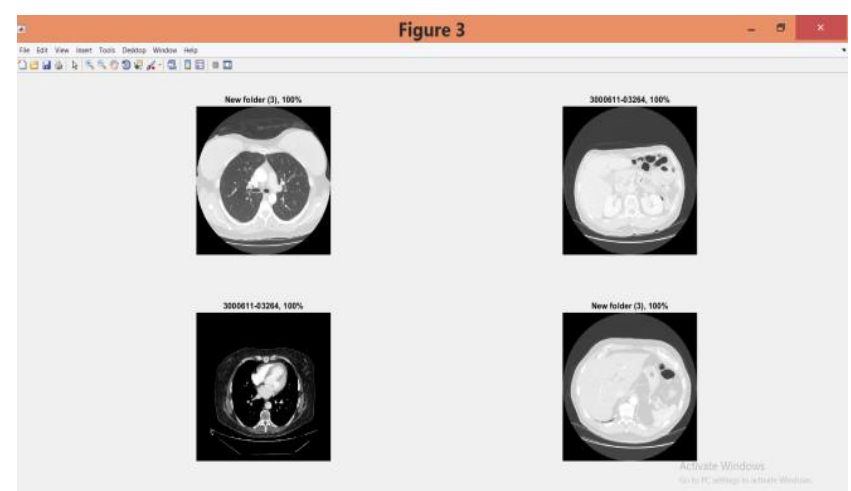

Figure 10: Problem Identification using AlexNet

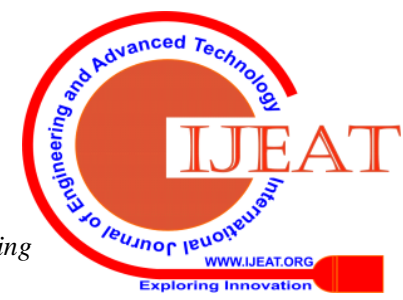




\section{A Deep Analysis of Google Net and AlexNet for Lung Cancer Detection}

\section{EXPERIMENTAL RESULTS AND DISCUSSION}

GoogleNet and AlexNet network are trained using MATLAB R2018b with a GPU system with the following specifications. Processor: Intel ${ }^{\circledR}$ Core $^{\mathrm{TM}}$ i7-8700 CPU @3.2GHZ and Graphics Card: NVIDIA GeForce GTX 1060 6GB. Several parameters are analyzed to compare the performance of GoogleNet and AlexNet.

Table-1: presents the mathematical values to compare both the networks.

\begin{tabular}{|l|l|l|l|l|l|l|}
\hline $\begin{array}{l}\text { Network } \\
\text { type }\end{array}$ & Time & Accuracy & Batch size & $\begin{array}{l}\text { No. of } \\
\text { Iterations }\end{array}$ & $\begin{array}{l}\text { Initial } \\
\text { Learning } \\
\text { Rate }\end{array}$ & Layers \\
\hline AlexNet & $20 \mathrm{Hrs}$ & $99.91 \%$ & 10 & 1620 & 0.0001 & 8 \\
\hline GoogleNet & $46 \mathrm{Hrs}$ & $99.05 \%$ & 10 & 1620 & 0.0001 & 22 \\
\hline
\end{tabular}

Table -1: Comparative Analysis on GoogleNet and AlexNet architecture for Fabric Defect Detection

The inputs that can be given to the network produces different outputs based on the method applied. GoogleNet and AlexNet are the networks that detects the lung cancer for the input size of 2705 images of LIDC-IDRI database with 1010 cases. GoogleNet determines the cancer in the lungs with accuracy rate of $99.05 \%$ with an elapsed time of about 46 hours,27sec. The AlexNet detects the lung cancer with 99.91\% accuracy rate with an elapsed time of 20 hours,11sec. Initially, the learning rate of the network is 0.0001 . The learning rate 0.0001 provides the maximum accurate results. For different learning rates, different parameters will vary and provides diverse outputs. If the learning rate of network is greater than 0.0001 ,training of images takes lesser time. The GoogleNet has 22 layers in deep which enhances the accuracy.The disadvantage of GoogleNet is - it increases the over-fitting problem. It can be reduced by data augmentation. The parameters like 'Initial learning rate', iterations, training time, layers increases the accuracy.

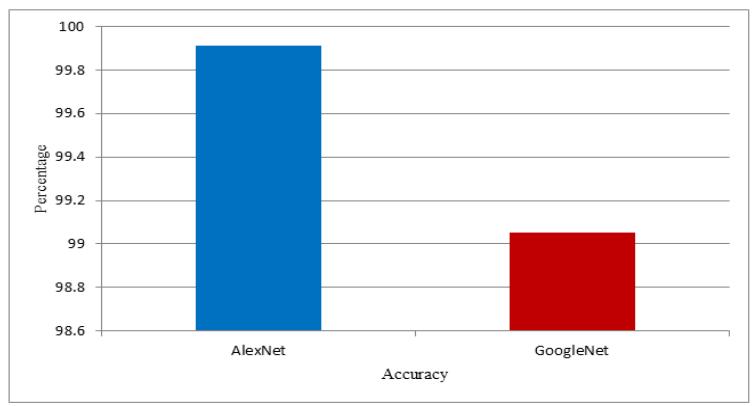

[a]

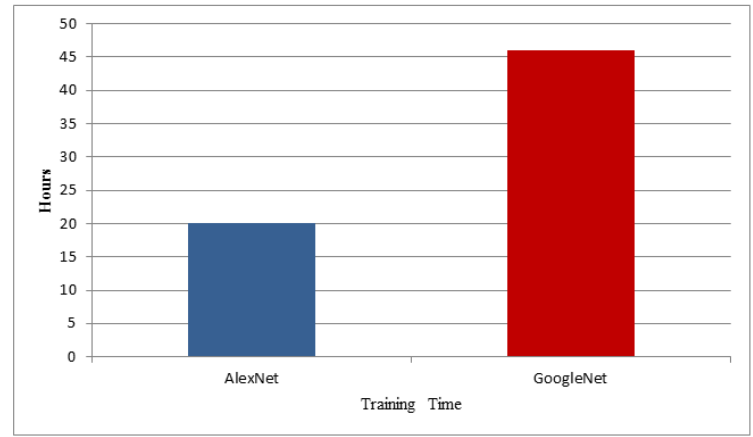

[b]

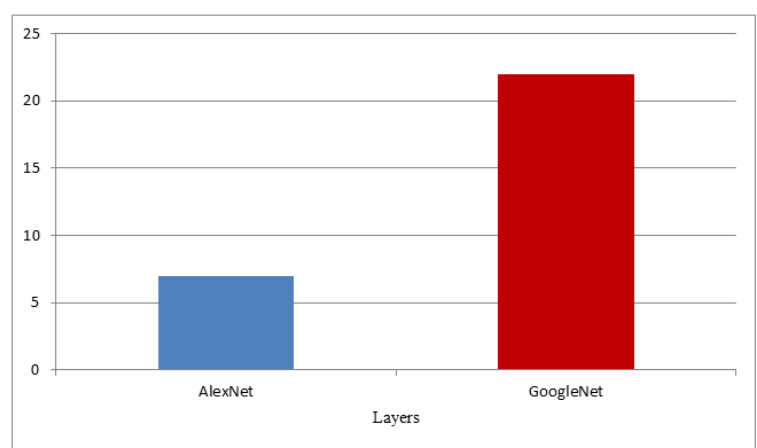

[c]

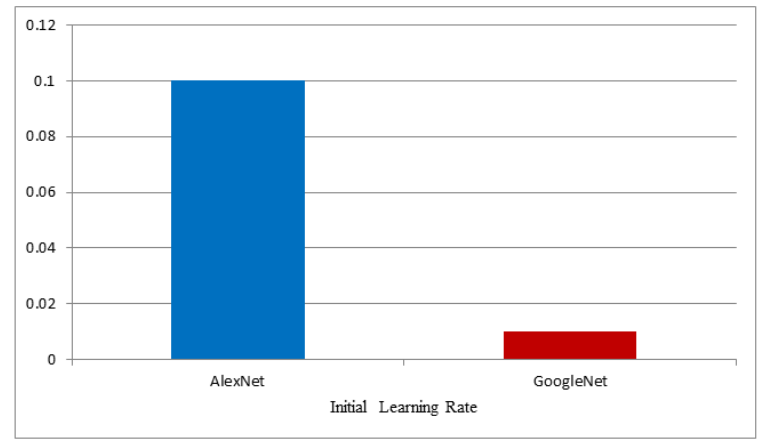

[d]

When compared to GoogleNet, AlexNet provides the better performance in training time and accuracy. AlexNet is modelled in bigger size provided with 7 hidden layers, $650 \mathrm{~K}$ units and 60M parameters. Mainly the AlexNet uses the ReLU function for training the images. By this function, it provides the faster access and ReLU is put after every convolutional and FC layers. The main advantage of AlexNet is reduces the over-fitting problems by using the dropout layer. In this Network the training time is doubled with dropout ratio of 0.5[11]. AlexNet is more efficient network in order to recognize the lung cancer with 99.91 percentage, accurate results are obtained. Then further treatments can be provided to the patients for their recovery.

\section{CONCLUSION}

Obviously, AlexNet provides the better results than that of GoogleNet as the deep learning methods for the detection of lung cancer. Many authors assisted to the deep learning subject in determining the lung cancer by analyzing various existing surveys. GoogleNet and AlexNet are deep learning networks that can be employed for cancer detection. Both of the networks do a great job in detecting the problem,but AlexNet's performance out-weights than that of GoogleNet with respect to various parameters. The results indicate that former is better than that of the latter one considering the parameters like accuracy, learning rate, training time, maximum iterations. AlexNet can be given first preference in lung cancer detection. In future, these networks can be improved further to detect the lung cancer at earlier stage and increase the 5 year survival rate of the affected people. 


\section{REFERENCES}

1. KeyStatisticsforLungCancerhttps://www.cancer.org/cancer/nonsmallc elllungcancer/about/keystatistics.html

2. https://www.who.int/newsroom/factsheets/detail/cancer

3. A.C.Society,CancerFactsandFigures,2015,http://www.cancer.org/acs/ groups/content/@editorial/documents/docum ent/acspc-044552.pdf.

4. I.R.Valente, P.C.Cortez, E.C.Neto, J.M.Soares, V. H.de Albuquerque, and J.M.Tavares, "Automatic 3D pulmonary nodule detection in CT images: a survey," Computer Methods and Programs in Biomedicine, vol.124,no.1,pp.91-107,2016.

5. Shuang Mei, Yudan Wang, and Guojun Wen. (2 Apr 2018) "Automatic Fabric Defect Detection with a Multi-Scale Convolutional Denoising Autoencoder Network Model", Sensors, pp 1-18.

6. Zhu, Z.Wang, X. Bai, S. Yao, C. Bai, X. (2016). "Deep learning representation using autoencoder for $3 \mathrm{D}$ shape retrieval", Neuro-computing, vol 204, pp 41-50

7. Umit V.C, ataly urek, Cevdet Aykanat, and Bora Uc,ar. On two-dimensional sparse matrix par- " titioning: Models, methods, and a recipe. SIAM J. Sci. Comput., 32(2):656-683, February 2010.

8.

http://web.cse.ohiostate.edu/ panda.2/5194/slides/2.c.GoogLeNet.pdf (accessed on 3rd April 2019)

9. $\quad$ Bell S, Zitnick C L, Bala K,et. (2016).“Inside-outside net: Detecting objects in context with skip pooling and recurrent neural networks",Computer Vision and Pattern Recognition, pp 1-11

10. MATLABAvailableonlinehttps://www.mathworks.com/help/matlab/r ef/matlab.io.datastore.imagedatastore.split-each-label.html(accessed on 10th March 2019).

11. https://medium.com/@RaghavPrabhu/cnnarchitectureslenetalexnetvgg googlenetandresnet7c81c017b848

\section{AUTHORS PROFILE}

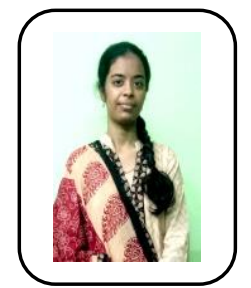

B.ALMAS, received her Bachelor's Degree in Electronics and Communication, at Madanapalle Institute of Technology \& Science, Madanapalle, Andhra Pradesh in 2018.Currently Pursuing her M.Tech in Digital Electronics \& Communication Engineering in Madanapalle Institute of Technology \& Science, Madanapalle, Andhra Pradesh. Her current research interests include Deep Learning and Image Processing.

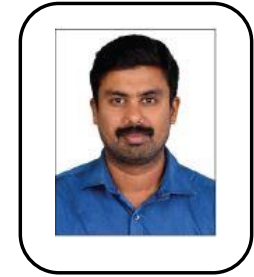

Learning.

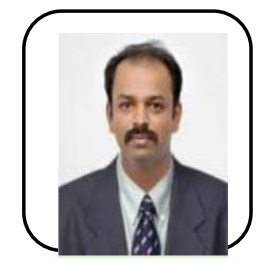

S. RAJASEKARAN completed B.E., M.E and Ph.D. from Anna University, Chennai in 2003, 2007 and 2014 respectively. He has the total experience of 15 years in teaching field. Currently working as, a Associate Professor in Department of ECE at Madanapalle Institute of Technology \& Science, Madanapalle, Andhra Pradesh. His current research interests include Biomedical Image Processing, Deep Learning \& Control Systems.

K. SATHESH completed B.E., M.E and Ph.D. from Anna University, Chennai in 2006, 2008 and 2016 respectively. Joined as a Lecturer in the year 2008 and has the total experience of 12 years in teaching field. Currently working as, a Associate Professor in ECE at Madanapalle Institute of Pradesh. His current research interests include Signal Processing, Biomedical Image Processing \& Deep 